\title{
A Research on the Determination of Phenological and Molecular Characterization in Open-pollinated Genotypes in Walnut
}

\section{Mehmet Sütyemez}

Kahramanmaraş Sütçü İmam Üniversitesi: Kahramanmaras Sutcu Imam Universitesi

selma BOYACI ( $\nabla$ selma.boyaci@ahievran.edu.tr)

Kirsehir Ahi Evran Universitesi https://orcid.org/0000-0002-3349-839X

\section{Nur Kuscuoglu}

Kahramanmaras Sutcu Imam Universitesi - Avsar Kampusu: Kahramanmaras Sutcu Imam Universitesi

\section{Akide Ozcan}

Kahramanmaras Sutcu Imam Universitesi - Avsar Kampusu: Kahramanmaras Sutcu Imam Universitesi

\section{Sakir Burak Bukucu}

Selçuk Üniversitesi: Selcuk Universitesi

\section{Esra Yıldırım}

Kahramanmaras Sutcu Imam University: Kahramanmaras Sutcu Imam Universitesi

\section{Adem Bardak}

Kahramanmaras Sutcu Imam University: Kahramanmaras Sutcu Imam Universitesi

\section{Research Article}

Keywords: Juglans regia L., molecular, phenology, ISSR, variation

Posted Date: June 21st, 2021

DOl: https://doi.org/10.21203/rs.3.rs-605698/v1

License: (c) (i) This work is licensed under a Creative Commons Attribution 4.0 International License. Read Full License

Version of Record: A version of this preprint was published at Harran Tarım ve Gıda Bilimleri Dergisi on December 25th, 2021. See the published version at https://doi.org/10.29050/harranziraat.986501. 
Key words: Juglans regia L., molecular, phenology, ISSR, variation

\section{INTRODUCTION} 46040, TURKEY 46650, Kahramanmaras, TURKEY Tasucu, Mersin, TURKEY Corresponding author: selma.boyaci@ahievran.edu.tr

\section{ABSTRACT} significant variation both phenologically and genetically.

\section{A RESEARCH ON THE DETERMINATION OF PHENOLOGICAL AND MOLECULAR CHARACTERIZATION IN OPEN-POLLINATED GENOTYPES IN WALNUT}

${ }^{1}$ Department of Horticulture, Agricultural Faculty, Kahramanmaras Sutcu Imam University, Kahramanmaras

${ }^{2}$ Department of Horticulture, Agricultural Faculty, Kirşehir Ahi Evran University, Kirşehir 40100, TURKEY

${ }^{3}$ Department of Organic Farming, Goksun Vocational School, Kahramanmaras Sutcu Imam University, Goksun

${ }^{4}$ Department of Plant and Animal Production, Silifke Tasucu Vocational School, Selcuk University, 33900 Silifke

${ }^{5}$ Department of Agricultural Biotechnology Kahramanmaras Sutcu Imam University Kahramanmaras TURKEY

This research was carried out to reveal the phenological and genetic differences between the S-1/1 walnut genotype and $94 \mathrm{~F} 1$ genotypes obtained from this genotype with each other and with the maternal parent. In the phenological observations made, it was observed that bud burst in genotypes took 52 days, leafing 50 days, leaf yellowing 31 days, and defoliation date 27 days. When the maternal parent (S-1/1) and the genotypes were compared, it was found that there was a phenological variation of $75.54 \%$ in budburs $t, 73.41 \%$ in the leafing, $34.05 \%$ in leaf yellowing, and $93.62 \%$ in defoliation date, while the average variation was $69.15 \%$. In molecular genetic analyzes, 7 ISSR primers were used to determine genetic variations, as a result, 7 monomorphic and 45 polymorphic bands were obtained, and the rate of polymorphism was found to be $86.53 \%$. The average number of alleles was calculated to be 7.42. In genotypes, the polymorphism information content (PIC) value varied between 0.48 and 0.95 , while the average PIC value was calculated to be 0.73 . As a result of cluster analysis, it was seen that genotypes were divided into 2 main clusters and 2 subsets. At the end of the study, it was determined that the S-1/1 walnut genotype and F1 genotypes obtained from this genoty pe have a

Since walnut is monoecious, has a tendency to dichogamy and has a heterozygous structure, seedlings do not show the same characteristics as the main variety when propagated by seed. This population is a great source of genes for breeders, while causes economic losses in domestic and foreign markets, as well as inability to provide standardization for breeders. A long period of time (10-15 years) is needed for characterization of a variety by selection and hybridization breeding in walnuts. Biotechnological methods recently were used to decrease the 
time needed for characterization. The use of these methods enables the characterization of genetic populations grown in different ecologies in a shorter time. Molecular marker techniques allow measuring biotechnological DNA levels and tracking the desired gene. The marker techniques are used in many areas related to ge nes such as phylogenetic analysis, mapping, and new gene discoveries (Filiz and Koç 2011). Reliability and practical use of ISSR markers and the length of primers provide advantages. The use of ISSR primers, which will deliver the desired information, is preferred due to the less time, labor and cost. The ISSR primers recently have been widely used to determine the genetic diversity in many plant species, phylogenetic studies and reveal genome maps (Yorgancilar et al. 2015).

Very few studies have been carried out to determine the phenological and genetic variation of the seedling genotypes obtained from the walnut seed compared to the main variety (Sutyemez et al. 2018; Bukucu et al. 2020; Ozcan et al. 2020; Ylldırm and Sütyemez 2020). Therefore, the studies investigating the phenological and genetic variation in F1 genotypes obtained especially from varieties to be used as main parents, are important to provide valuable information for crossbreeding breeding studies.

The purpose of this study was to determine the phenological and genetic relationships between the F1 plants obtained from the open-pollinated seeds of the S-1/1 walnut genotype with very high fruit size and the main parent variety.

\section{MATERIAL AND METHOD}

\section{2.1. Plant Material}

In the study, S-1/1 walnut genotype selected by Sütyemez (2019) and 94 seedling genotypes (F1) obtained from open-pollinated seeds belonging to this genotype were used.

Important characteristics of S1/1 genotype are highly productive and early, fruit weight ranges from 23 and $24 \mathrm{~g}$, kernel ratio is between 50 and $52 \%$, kernel color is light, outer shell is clear and smooth.

\subsection{Phenological observations}

62 The phenological traits such as budburst, leafing, leaf yellowing and defoliation dates of the main genotype S$631 / 1$ and other walnut genotypes were defined according to Walnut Descriptors (Table 1). The classification of phenological characteristics was given in Table 2.

65 Table 1. Definitions used to determine the phenological traits (IPGRI 1994)

\begin{tabular}{|l|l|}
\hline Traits & Description \\
\hline Budburst & $\begin{array}{l}\text { When over } 50 \% \text { of terminal buds have enlarged and the bud scales have split to } \\
\text { expose the inside green leaves }\end{array}$ \\
\hline Leafing & $\begin{array}{l}\text { When over } 50 \% \text { of terminal buds have enlarged and the bud scales have split } \\
\text { exposing the green leaves }\end{array}$ \\
\hline Leaf yellowing & When more than $50 \%$ of the green leaves on the plant turn yellow \\
\hline Defoliation & When all the leaves of the plant have fallen \\
\hline
\end{tabular}


Table 2. Classification of phenological characteristics of genotypes according to the main cultivar

\begin{tabular}{|l|l|}
\hline Clas sification & Phenological similarity to main cultivar (in days) \\
\hline Very close & $\pm 0-3$ \\
\hline Close & $\pm 4-8$ \\
\hline Far & $\pm 9-14$ \\
\hline Very Far & $\geq \pm 15$ \\
\hline
\end{tabular}

67 Source: Sütyemez (2018), + after, - before

\section{2.3. Phenological data analysis}

69 Descriptive statistics, cluster analyses, Principal Component Analyses (PCA) and correlation test were carried 70 out using the JMP13 statistical software to reveal the information on genetic diversity. Phenological pair-wise 71 distances of the walnut genotypes were clustered using Ward's method (Anderberg 1973).

\subsection{Molecular analyzes}

\section{DNA isolation and extraction}

The leaflets of S-1/1 genotype and leaflets 94 seedlings obtained from S-1/1 genotype were collected in the spring and brought to the laboratory in dry ice. The young and healthy leaves samples were passed through $70 \%$ alcohol and distilled water and kept at $-80{ }^{\circ} \mathrm{C}$ until DNA isolation. The DNA isolation of leaf samples was performed using the CTAB protocol developed by Doyle Doyle (1987) and modified by Bardak (2012). In order to obtain gel images of the S-1/1 walnut genotypes; $3 \%$ agarose gel was prepared and the DNAs were run in $1 \mathrm{x}$ TBE buffer solution at 160 voltage electric current for 2 hours. After running, the gel was treated with ethidium bromide ( 1 liter distilled water $+300 \mu$ ethidium bromide) for 15 minutes. Stained DNA bands were visualized under UV (ultraviolet) light.

\section{ISSR-PCR amplification}

83 Twelve ISSR primers were prepared using the ISSR technique developed by Zietkiewicz et al. (1994) and 84 modified by Bardak and Bölek (2012) to determine the genetic similarity rates between 94 S-1/1 genotypes. The 85 ISSR reading range of PCR analysis for 94 walnut genotypes is between 200 and 1000 base pairs. In the scoring, 86 DNA bands of walnut genotypes and main variety were compared, and the bands of the same length were evaluated as ' 1 ', while bands of different lengths were evaluated as ' 0 ' (Zhang et al. 2005; Bardak and Bölek 2012).

\section{Data analysis}

90 The polymorphism information contents of the primers used in the study were calculated in Excel using the data 91 obtained by scoring (Laborda et al. 2005). The sums of ' 1 ' and ' 0 ' allele scores from the polymorphic bands were obtained and the allele frequencies of each band were calculated using the following equation;

94 Where, $f$ I represents the frequency of $\mathrm{i}$ band.

95 Genetic distance was determined using POPGENE32 (Population Genetic Analysis; version 1.32) software 96 according to Nei (1972). The phylogenetic tree was obtained in the MEGA 7 (Molecular Evolutionary Genetics 

genetic distance data.

99 Cluster analysis of S-1/1 walnut genotypes was performed using Structure 2.3.4 software. Each K value was run

100 from 1 to 10 with 10 independent simulations to determine the ideal number of groups.

101 The permutation module was chosen between 10000 and 100000 and the $\Delta \mathrm{K}$ value, which determines the 102 number of groups, was calculated by 5 replications for each $\mathrm{K}$ value. The results of analysis were archived in a 103 zip file and the ideal $\Delta \mathrm{K}$ value was determined by uploading this file to the "structure harvester" web page 104 (http://taylor0.biology.ucla.edu/struct ureHarvester/).

\section{3. RESULTS AND DISCUSSION}

106 Late spring and early autumn frosts cause the most damage in fruit growing (A ğaoğlu et al. 2019). Therefore, the

107 first leafing and defoliation dates of fruit species are important. The walnut is adversely affected by late spring 108 and early autumn frosts, therefore, obtaining genotypes with late leafing and early defoliation is one of the main 109 objectives in breeding studies (Özcan et al. 2020).

\section{$110 \quad 3.1$ Phenologic Observations}

111 Phenological variation was determined between the S-1/1 main cultivar and the seedlings obtained from open-

112 pollinated seeds of S-1/1 offspring genotypes (Table 3; Table 4).

113 The budburst date of the S-1/1 main cultivar occurred on March 16, while the budburst of F1 genotypes occurred 114 in a 52-day period. Twenty three of F1 genotypes were classified (24.46\%) in the "very close" category to main 115 cultivar, 44 genotypes (46.8\%) were in the "close" category, 24 genotypes (25.53\%) were in the "far" category, 116 and 3 genotypes (3.19\%) were in the "very far" category (Table 4). Phenological variation between the F1 117 genotypes and the main cultivar as indicated by budburst date was $75.54 \%$. The mean number of days of 118 variation ratio between S-1/1 and the other 94 genotypes was 4.52, and the period ranged from 0 to 41 days 119 (Table 4).

120 The first leafing date in F1 genotypes was in a wide range of 50 days. The number of F1 genotypes classified in 121 "very close" category in terms of first leafing was 25 (26.46\%), 26 (22.67\%) of F1 genotypes were classified in 122 "close" category, $21(22.34 \%)$ of them were in "far" category, and $22(23.4 \%)$ of them were "very far" 123 category. A phenological variation of $73.41 \%$ was detected between the main cultivar and the F1 genotypes in 124 terms of the first leafing.

125 The average variation in days between the main genotype and the F1 genotypes was 8.7, and the period was 126 between 0-44 days (Table 4). The leaf yellowing date of main cultivar was November 4, and the leaf yellowing 127 start time of the 9 F1 genotypes was spread over 26 days. The leaf yellowing dates of the 62 F1 genotypes (65.95 $128 \%)$ were "very close" to the main genotype, 18 genotypes (19.14\%) were "close", 10 genotypes (10.63\%) were 129 "far" and 4 genotypes (4.25 \%) were "very far". The phenological variation as indicated by the onset of leaf 130 yellowing between the main cultivar and F1 genotypes (32 genotypes) was 34.05\%. The mean number of days of 131 variation between S-1/1 and $94 \mathrm{~F} 1$ genotypes was 1.52, and the date of onset of yellowing ranged from 0 to 24 132 days (Table 4). 
133 The defoliation in the S-1/1 main genotype occurred on November 15, while the defoliation dates of 94 134 genotypes were spread over 43 days. The defoliation date of $6(6.38 \%)$ seedling genotypes was "very close" to 135 the main cultivar, 31 (32.97\%) of genotypes were "close", 31 (32.97 \%) of them were "far" and 26 (27.65\%) of 136 them were "very far" to main cultivar. The phenological variation between the main cultivar and the other 137 genotypes (with 88 genotypes) in terms of defoliation date was calculated as $93.62 \%$. The average number of 138 days of variation ratio between S-1/1 and $94 \mathrm{~F} 1$ genotypes was $11.21 \%$, and the date of defoliation ranged from 1390 to 33 days (Table 4). 
Table 3. Some phenological characteristics and classification of walnut genotypes (According to S-1/1)

\begin{tabular}{|c|c|c|c|c|c|c|c|c|c|c|c|c|}
\hline Genotypes & Budburst & $\begin{array}{c}\text { From S-1/1 } \\
\text { (days) }\end{array}$ & Classification & First leafing & \begin{tabular}{|c}
$\begin{array}{c}\text { From S-1/1 } \\
\text { (days) }\end{array}$ \\
\end{tabular} & Classification & Leaf yellowing & \begin{tabular}{|c}
$\begin{array}{c}\text { From S-1/1 } \\
\text { (days) }\end{array}$ \\
\end{tabular} & Classification & Defoliation & $\begin{array}{c}\text { From S-1/1 } \\
\text { (days) }\end{array}$ & Classification \\
\hline Genotype 1 & $22 / 03$ & +6 & Close & $31 / 03$ & +10 & Far & $06 / 11$ & +2 & Very Close & $13 / 12$ & +28 & Very Far \\
\hline Genotype 2 & $20 / 03$ & +4 & Close & $28 / 03$ & +7 & Close & $04 / 11$ & 0 & Very Close & $13 / 12$ & +28 & Very Far \\
\hline Genotype 3 & $11 / 03$ & -5 & Close & $22 / 03$ & +1 & Very Close & $04 / 11$ & 0 & Very Close & $17 / 12$ & +32 & Very Far \\
\hline Genotype 4 & $09 / 03$ & -7 & Close & $18 / 03$ & -3 & Very Close & $03 / 11$ & -1 & Very Close & $29 / 11$ & +14 & Far \\
\hline Genotype 5 & $15 / 03$ & -1 & Very Close & $23 / 03$ & +2 & Very Close & $05 / 11$ & +1 & Very Close & $28 / 11$ & +13 & Far \\
\hline Genotype 6 & $19 / 03$ & +3 & Very Close & $28 / 03$ & +7 & Close & $04 / 11$ & 0 & Very Close & $13 / 12$ & +28 & Very Far \\
\hline Genotype 7 & $27 / 03$ & +11 & Far & $8 / 04$ & +18 & Very Far & $09 / 11$ & +5 & Close & $28 / 11$ & +13 & Far \\
\hline Genotype 8 & 08/03 & -8 & Close & $17 / 03$ & -4 & Close & $31 / 10$ & -4 & Close & $29 / 11$ & +14 & Far \\
\hline Genotype 9 & $13 / 03$ & -3 & Very Close & $21 / 03$ & 0 & Very Close & $12 / 11$ & +8 & Close & $02 / 12$ & +4 & Close \\
\hline Genotype 10 & $16 / 03$ & 0 & Very Close & $23 / 03$ & +2 & Very Close & $03 / 11$ & -1 & Very Close & $14 / 12$ & +29 & Very Far \\
\hline Genotype 11 & $21 / 03$ & +5 & Close & $02 / 04$ & +12 & Far & $19 / 11$ & +15 & Far & $15 / 12$ & +30 & Very Far \\
\hline Genotype 12 & $26 / 03$ & +10 & Far & $06 / 04$ & +16 & Very Far & $08 / 11$ & -4 & Close & $03 / 12$ & +18 & Very Far \\
\hline Genotype 13 & $27 / 03$ & +11 & Far & $06 / 04$ & +16 & Very Far & $13 / 11$ & +9 & Far & $17 / 12$ & +32 & Very Far \\
\hline Genotype 14 & $23 / 03$ & +7 & Close & $01 / 04$ & +11 & Far & $07 / 11$ & +3 & Very Close & $18 / 12$ & +33 & Very Far \\
\hline Genotype 15 & $13 / 03$ & -3 & Very Close & $21 / 03$ & 0 & Very Close & $05 / 11$ & +1 & Very Close & $28 / 11$ & +13 & Far \\
\hline Genotype 16 & $11 / 03$ & -5 & Close & $20 / 03$ & -1 & Very Close & $03 / 11$ & -1 & Very Close & $06 / 12$ & +21 & Very Far \\
\hline Genotype 17 & $26 / 03$ & +10 & Far & $02 / 04$ & +12 & Far & $02 / 11$ & -2 & Very Close & $23 / 11$ & +8 & Close \\
\hline Genotype 18 & $02 / 04$ & +17 & Very Far & $08 / 04$ & +18 & Very Far & $12 / 11$ & +8 & Close & $28 / 11$ & +13 & Far \\
\hline Genotype 19 & $28 / 03$ & +12 & Far & $07 / 04$ & +17 & Very Far & $31 / 10$ & -4 & Close & $13 / 11$ & -2 & Very Close \\
\hline Genotype 20 & $30 / 03$ & +14 & Far & $08 / 04$ & +18 & Very Far & $02 / 11$ & -2 & Very Close & $28 / 11$ & +13 & Far \\
\hline Genotype 21 & $26 / 04$ & +41 & Very Far & 03/04 & +43 & Very Far & $30 / 10$ & -5 & Close & $28 / 11$ & +13 & Far \\
\hline Genotype 22 & $29 / 03$ & +13 & Far & $07 / 04$ & +17 & Very Far & $03 / 11$ & -1 & Very Close & $09 / 11$ & -6 & Close \\
\hline Genotype 23 & $28 / 03$ & +12 & Far & $08 / 04$ & +18 & Very Far & $03 / 11$ & -1 & Very Close & $18 / 11$ & +3 & Very Close \\
\hline
\end{tabular}




\begin{tabular}{|c|c|c|c|c|c|c|c|c|c|c|c|c|}
\hline Genotypes & Budburst & $\begin{array}{c}\text { From S-1/1 } \\
\text { (days) }\end{array}$ & Classification & First leafing & \begin{tabular}{|c}
$\begin{array}{c}\text { From S-1/1 } \\
\text { (days) }\end{array}$ \\
\end{tabular} & Classification & $\begin{array}{c}\text { Leaf } \\
\text { Yellowing }\end{array}$ & $\begin{array}{c}\text { From S-1/1 } \\
\text { (days) }\end{array}$ & Classification & Defoliation & $\begin{array}{c}\text { From S-1/1 } \\
\text { (days) }\end{array}$ & Classification \\
\hline Genotype 24 & $12 / 03$ & -4 & Close & $20 / 03$ & -1 & Very Close & $05 / 11$ & +1 & Very Close & $21 / 11$ & +6 & Close \\
\hline Genotype 25 & $19 / 03$ & +3 & Very Close & $25 / 03$ & +4 & Close & $03 / 11$ & -1 & Very Close & $20 / 11$ & +5 & Close \\
\hline Genotype 26 & $5 / 03$ & -11 & Far & $17 / 03$ & -4 & Close & $31 / 10$ & -4 & Close & $28 / 11$ & +13 & Far \\
\hline Genotype 27 & $21 / 03$ & +5 & Close & $27 / 03$ & +6 & Close & $12 / 11$ & +8 & Close & $28 / 11$ & +13 & Far \\
\hline Genotype 28 & $21 / 03$ & +5 & Close & $29 / 03$ & +8 & Close & $05 / 11$ & +1 & Very Close & $21 / 11$ & -6 & Close \\
\hline Genotype 29 & $20 / 03$ & +4 & Close & $28 / 03$ & +7 & Close & $05 / 11$ & +1 & Very Close & $24 / 11$ & +9 & Far \\
\hline Genotype 30 & $21 / 03$ & +5 & Close & $29 / 03$ & +8 & Close & $04 / 11$ & 0 & Very Close & $11 / 11$ & -4 & Close \\
\hline Genotype 31 & $20 / 03$ & +4 & Close & $26 / 04$ & +5 & Close & $01 / 11$ & +1 & Very Close & $25 / 11$ & +10 & Far \\
\hline Genotype 32 & $11 / 03$ & -5 & Close & $19 / 03$ & -2 & Very Close & 05/11 & +1 & Very Close & $25 / 11$ & +10 & Far \\
\hline Genotype 33 & $17 / 03$ & +3 & Very Close & $26 / 03$ & +5 & Close & $05 / 11$ & +1 & Very Close & $23 / 11$ & +9 & Far \\
\hline Genotype 34 & $18 / 03$ & +2 & Very Close & $25 / 03$ & +4 & Close & $19 / 11$ & +15 & Far & $29 / 11$ & +14 & Far \\
\hline Genotype 35 & $24 / 03$ & +8 & Close & $02 / 04$ & +12 & Far & $02 / 11$ & -2 & Very Close & $10 / 11$ & -5 & Close \\
\hline Genotype 36 & $16 / 03$ & 0 & Very Close & $22 / 03$ & +1 & Very Close & $29 / 10$ & -6 & Close & $10 / 11$ & -5 & Close \\
\hline Genotype 37 & 08/03 & -8 & Close & $20 / 03$ & -1 & Very Close & $03 / 11$ & -1 & Very Close & $11 / 11$ & -6 & Close \\
\hline Genotype 38 & $22 / 03$ & +6 & Close & $01 / 04$ & +11 & Far & $02 / 11$ & -2 & Very Close & $20 / 11$ & +5 & Close \\
\hline Genotype 39 & $21 / 03$ & +5 & Close & $31 / 03$ & +10 & Far & $03 / 11$ & -1 & Very Close & $10 / 11$ & -5 & Close \\
\hline Genotype 40 & $30 / 03$ & +14 & Far & $10 / 04$ & +20 & Very Far & $31 / 10$ & -4 & Close & $29 / 11$ & +14 & Far \\
\hline Genotype 41 & $21 / 03$ & +5 & Close & $01 / 04$ & +11 & Far & $03 / 11$ & -1 & Very Close & $24 / 11$ & +9 & Far \\
\hline Genotype 42 & $21 / 03$ & +5 & Close & $30 / 03$ & +9 & Far & $05 / 11$ & +1 & Very Close & $08 / 11$ & -7 & Close \\
\hline Genotype 43 & $24 / 03$ & +8 & Close & 03/04 & +13 & Far & $03 / 11$ & -1 & Very Close & $11 / 11$ & -4 & Close \\
\hline Genotype 44 & $23 / 03$ & +7 & Close & $01 / 04$ & +11 & Far & $04 / 11$ & 0 & Very Close & $11 / 11$ & -4 & Close \\
\hline Genotype 45 & $22 / 03$ & +6 & Close & $30 / 03$ & +9 & Far & $02 / 11$ & -2 & Very Close & $3 / 12$ & +18 & Very Far \\
\hline Genotype 46 & $19 / 03$ & +3 & Very Close & $26 / 03$ & +5 & Close & $02 / 11$ & -2 & Very Close & $10 / 11$ & -5 & Close \\
\hline Genotype 47 & $19 / 03$ & +3 & Very Close & $27 / 03$ & +6 & Close & 01/11 & -3 & Very Close & $18 / 11$ & +3 & Very Close \\
\hline Genotype 48 & $12 / 03$ & -4 & Close & $21 / 03$ & 0 & Very Close & $31 / 10$ & -4 & Close & $25 / 11$ & +10 & Far \\
\hline
\end{tabular}




\begin{tabular}{|c|c|c|c|c|c|c|c|c|c|c|c|c|}
\hline Genotype & Budburst & \begin{tabular}{|c}
$\begin{array}{c}\text { From S-1/1 } \\
\text { (days) }\end{array}$ \\
\end{tabular} & Classification & First leafing & \begin{tabular}{|c|}
$\begin{array}{c}\text { From S-1/1 } \\
\text { (days) }\end{array}$ \\
\end{tabular} & Classification & Leaf Yellowing & \begin{tabular}{|c}
$\begin{array}{c}\text { From S-1/1 } \\
\text { (days) }\end{array}$ \\
\end{tabular} & Classification & \begin{tabular}{|l|} 
Defoliation \\
\end{tabular} & $\begin{array}{c}\begin{array}{c}\text { From S-1/1 } \\
\text { (days) }\end{array} \\
\end{array}$ & Classification \\
\hline Genotype 49 & $18 / 03$ & +2 & Very Close & $24 / 03$ & +3 & Very Close & $02 / 11$ & -2 & Very Close & $05 / 11$ & +10 & Far \\
\hline Genotype 50 & $24 / 03$ & +8 & Close & $02 / 04$ & +12 & Far & $04 / 11$ & 0 & Very Close & $18 / 11$ & +13 & Far \\
\hline Genotype 51 & $21 / 04$ & +5 & Close & $04 / 05$ & +44 & Very Far & $03 / 11$ & -1 & \begin{tabular}{|l} 
Very Close \\
\end{tabular} & $24 / 11$ & +9 & Far \\
\hline Genotype 52 & $20 / 03$ & +4 & Close & $27 / 03$ & +6 & Close & 03/11 & -1 & Very Close & $10 / 11$ & -5 & Close \\
\hline Genotype 53 & $12 / 03$ & -4 & Close & $21 / 03$ & 0 & Very Close & $26 / 10$ & -9 & Far & $19 / 11$ & +4 & Close \\
\hline Genotype 54 & $26 / 03$ & +10 & Far & $05 / 04$ & +15 & Far & $02 / 11$ & -2 & Very Close & $24 / 11$ & +9 & Far \\
\hline Genotype 55 & $27 / 03$ & +11 & Far & $06 / 04$ & +16 & Very Far & $02 / 11$ & -2 & Very Close & $18 / 11$ & +3 & Very Close \\
\hline Genotype 56 & $30 / 03$ & +14 & Far & $06 / 04$ & +16 & Very Far & $03 / 11$ & -1 & Very Close & $19 / 11$ & +4 & Close \\
\hline Genotype 57 & $26 / 03$ & +10 & Far & $06 / 04$ & +16 & Very Far & $03 / 11$ & -1 & Very Close & $28 / 11$ & +13 & Far \\
\hline Genotype 58 & $27 / 03$ & +11 & Far & $06 / 04$ & +16 & Very Far & $02 / 11$ & -2 & Very Close & $13 / 11$ & -2 & Very Close \\
\hline Genotype 59 & $12 / 03$ & -4 & Close & $21 / 03$ & +0 & Very Close & $12 / 11$ & +8 & Close & $28 / 11$ & +13 & Far \\
\hline Genotype 60 & $11 / 03$ & -5 & Close & $20 / 03$ & -1 & Very Close & $05 / 11$ & +1 & Very Close & $10 / 11$ & -5 & Close \\
\hline Genotype 61 & $13 / 03$ & -3 & Very Close & $27 / 03$ & +6 & Close & $30 / 10$ & -5 & Close & $19 / 11$ & +4 & Close \\
\hline Genotype 62 & $30 / 03$ & +14 & Far & $08 / 04$ & +18 & Very Far & $08 / 11$ & +4 & Close & $28 / 11$ & +13 & Far \\
\hline Genotype 63 & $17 / 03$ & +1 & Very Close & $23 / 03$ & +2 & Very Close & $31 / 10$ & -4 & Close & $20 / 11$ & +5 & Close \\
\hline Genotype 64 & $21 / 03$ & +5 & Close & $29 / 03$ & +8 & Close & $05 / 11$ & +1 & Very Close & $21 / 11$ & +6 & Close \\
\hline Genotype 65 & $15 / 03$ & -1 & Very Close & $24 / 03$ & +3 & Very Close & $02 / 11$ & -2 & Very Close & $10 / 11$ & -5 & Close \\
\hline Genotype 66 & $26 / 03$ & +10 & Far & $02 / 04$ & +12 & Far & $03 / 11$ & +22 & Very Far & $03 / 12$ & +18 & Very Far \\
\hline Genotype 67 & $24 / 03$ & +8 & Close & $02 / 04$ & +12 & Far & $18 / 11$ & +24 & Very Far & $18 / 12$ & +33 & Very Far \\
\hline Genotype 68 & $30 / 03$ & +14 & Far & $08 / 04$ & +18 & Very Far & $01 / 11$ & -3 & Very Close & $19 / 11$ & +4 & Close \\
\hline Genotype 69 & $21 / 03$ & +5 & Close & $29 / 03$ & +8 & Close & $02 / 11$ & -2 & Very Close & $23 / 11$ & +8 & Close \\
\hline Genotype 70 & $21 / 03$ & +5 & Close & $29 / 03$ & +8 & Close & $03 / 11$ & -1 & Very Close & $03 / 12$ & +18 & Very Far \\
\hline Genotype 71 & $11 / 03$ & -5 & Close & $20 / 03$ & -1 & Very Close & $04 / 11$ & 0 & Very Close & $28 / 11$ & +13 & Far \\
\hline Genotype 72 & $25 / 03$ & +9 & Far & $02 / 04$ & +12 & Far & $02 / 11$ & -2 & Very Close & $30 / 11$ & +15 & Far \\
\hline Genotype 73 & $31 / 03$ & +15 & Far & $08 / 04$ & +18 & Very Far & $05 / 11$ & +1 & Very Close & $28 / 11$ & +13 & Far \\
\hline
\end{tabular}




\begin{tabular}{|c|c|c|c|c|c|c|c|c|c|c|c|c|}
\hline Genotypes & Bud burst & $\begin{array}{c}\text { From S-1/1 } \\
\text { (days) }\end{array}$ & Classification & First leafing & $\begin{array}{c}\text { From S-1/1 } \\
\text { (days) }\end{array}$ & Classification & \begin{tabular}{c|} 
Leaf \\
Yellowing
\end{tabular} & $\begin{array}{c}\text { From S-1/1 } \\
\text { (days) }\end{array}$ & Classification & Defoliation & $\begin{array}{c}\text { From S-1/1 } \\
\text { (days) }\end{array}$ & Classification \\
\hline $\begin{array}{l}\text { Genotype } \\
74\end{array}$ & $16 / 03$ & 0 & Very Close & $23 / 03$ & +2 & Very Close & $13 / 11$ & +9 & Far & $13 / 12$ & +28 & Very Far \\
\hline Genotype 75 & $20 / 03$ & +4 & Close & $28 / 03$ & +7 & Close & $13 / 11$ & +9 & Far & $29 / 11$ & +14 & Far \\
\hline Genotype 76 & $21 / 03$ & +5 & Close & $30 / 03$ & +9 & Far & $02 / 11$ & -2 & Very Close & $19 / 11$ & +4 & Close \\
\hline Genotype 77 & $22 / 03$ & +6 & Close & $31 / 03$ & +10 & Far & $03 / 11$ & -1 & Very Close & $19 / 11$ & +4 & Close \\
\hline Genotype 78 & $21 / 03$ & +5 & Close & $29 / 03$ & +8 & Close & $04 / 11$ & 0 & Very Close & $19 / 11$ & +4 & Close \\
\hline Genotype 79 & $14 / 03$ & -2 & Very Close & $23 / 03$ & +2 & Very Close & $04 / 11$ & 0 & Very Close & $06 / 12$ & +21 & Very Far \\
\hline Genotype 80 & $27 / 03$ & +11 & Far & $06 / 04$ & +16 & Very Far & $04 / 11$ & 0 & Very Close & $04 / 12$ & +19 & Very Far \\
\hline Genotype 81 & $16 / 03$ & 0 & Very Close & $24 / 03$ & +3 & Very Close & $01 / 11$ & -3 & Very Close & $22 / 11$ & +7 & Close \\
\hline Genotype 82 & $19 / 03$ & +3 & Very Close & $26 / 03$ & +5 & Close & $04 / 11$ & 0 & Very Close & $14 / 11$ & -1 & Very Close \\
\hline Genotype 83 & $24 / 03$ & +8 & Close & $01 / 04$ & +11 & Far & $05 / 11$ & +1 & Very Close & $15 / 12$ & +30 & Very Far \\
\hline Genotype 84 & $7 / 03$ & -9 & Far & $18 / 03$ & -3 & Very Close & $03 / 11$ & -1 & Very Close & $28 / 11$ & +13 & Far \\
\hline Genotype 85 & $12 / 03$ & -4 & Close & $22 / 03$ & +1 & Very Close & $02 / 11$ & -2 & Very Close & $19 / 11$ & +4 & Close \\
\hline Genotype 86 & $25 / 03$ & +9 & Far & $06 / 04$ & +16 & Very Far & $04 / 11$ & 0 & Very Close & $28 / 11$ & +13 & Far \\
\hline Genotype 87 & $15 / 03$ & -1 & Very Close & $28 / 03$ & +7 & Close & $16 / 11$ & +12 & Far & $15 / 12$ & +30 & Very Far \\
\hline Genotype 88 & $22 / 03$ & +6 & Close & $02 / 04$ & +12 & Far & $12 / 11$ & +8 & Close & $12 / 12$ & +27 & Very Far \\
\hline Genotype 89 & $5 / 03$ & +11 & Far & $15 / 03$ & -6 & Close & $08 / 11$ & +4 & Close & $01 / 12$ & +16 & Very Far \\
\hline Genotype 90 & $4 / 04$ & +19 & Very Far & $12 / 04$ & +22 & Very Far & $13 / 11$ & +9 & Far & $15 / 12$ & +30 & Very Far \\
\hline Genotype 91 & $14 / 03$ & -2 & Very Close & $23 / 03$ & +2 & Very Close & $20 / 11$ & +16 & Very Far & $05 / 12$ & +20 & Very Far \\
\hline Genotype 92 & $15 / 03$ & -1 & Very Close & $29 / 03$ & +8 & Close & $18 / 11$ & +14 & Far & $04 / 12$ & +19 & Very Far \\
\hline Genotype 93 & $14 / 03$ & -2 & Very Close & $28 / 03$ & +7 & Close & $21 / 11$ & +17 & Very Far & $10 / 12$ & +25 & Very Far \\
\hline Genotype 94 & $23 / 03$ & +7 & Close & $08 / 04$ & +18 & Very Far & $14 / 11$ & +10 & Far & $12 / 12$ & +27 & Very Far \\
\hline$S-1 / 1$ & $16 / 03$ & 0 & - & $21 / 03$ & 0 & & $04 / 11$ & 0 & - & $15 / 11$ & 0 & - \\
\hline
\end{tabular}


Table 4. Phenological differences/similarities of S-1/1 walnut genotypes with the main cultivar

151

152 Late spring and early autumn frosts cause significant economic losses in walnuts. Therefore, the walnut

\begin{tabular}{|c|c|c|c|c|c|c|c|c|c|}
\hline \multirow[b]{2}{*}{$\begin{array}{c}\text { Phenologic } \\
\text { Characteristic }\end{array}$} & \multirow{2}{*}{$\begin{array}{c}\text { Variation } \\
\text { between } \mathrm{S}-1 / 1 \\
\text { and } 94 \\
\text { genotypes } \\
\text { (mean day) }\end{array}$} & \multirow[b]{2}{*}{$\begin{array}{l}\text { Belong } \\
\text { to } \mathrm{S}-1 / 1\end{array}$} & \multirow[b]{2}{*}{$\begin{array}{c}\text { First and last } \\
\text { date of } 94 \\
\text { genotypes }\end{array}$} & \multirow[b]{2}{*}{ Period } & \multicolumn{4}{|c|}{ Number of genotype (piece/\%) } & \multirow[b]{2}{*}{$\begin{array}{c}\text { Mean } \\
\text { variati } \\
\text { on }(\%)\end{array}$} \\
\hline & & & & & $\begin{array}{l}\text { Very } \\
\text { close }\end{array}$ & Close & Far & $\begin{array}{l}\text { Very } \\
\text { far }\end{array}$ & \\
\hline Bud Burst & $\begin{array}{r}4.52 \\
0-41\end{array}$ & 16.03 & $\begin{array}{l}05.03 \\
26.04\end{array}$ & 52 & $\begin{array}{c}23 \\
(24.46)\end{array}$ & $\begin{array}{c}44 \\
(46.8)\end{array}$ & $\begin{array}{c}24 \\
(25.53)\end{array}$ & $\begin{array}{c}3 \\
(3.19)\end{array}$ & 75.54 \\
\hline Leafing & $\begin{array}{c}8.7 \\
0-44\end{array}$ & 21.03 & $\begin{array}{l}15.03 \\
04.05\end{array}$ & 50 & $\begin{array}{c}25 \\
(26.46)\end{array}$ & $\begin{array}{c}26 \\
(22.67)\end{array}$ & $\begin{array}{c}21 \\
(22.34)\end{array}$ & $\begin{array}{c}22 \\
(23.4)\end{array}$ & 73.41 \\
\hline $\begin{array}{c}\text { Leaf } \\
\text { Yellowing }\end{array}$ & $\begin{array}{l}1.52 \\
0-24\end{array}$ & 4.11 & $\begin{array}{l}26.10 \\
21.11\end{array}$ & 27 & $\begin{array}{c}62 \\
(65.95)\end{array}$ & $\begin{array}{c}18 \\
(19.14)\end{array}$ & $\begin{array}{c}10 \\
(10.63)\end{array}$ & $\begin{array}{c}4 \\
(4.25)\end{array}$ & 34.05 \\
\hline Defoliation & $\begin{array}{l}11.21 \\
0-33\end{array}$ & 15.11 & $\begin{array}{l}05.11 \\
18.12 \\
\end{array}$ & 43 & $\begin{array}{c}6 \\
(6.38)\end{array}$ & $\begin{array}{c}31 \\
(32.97)\end{array}$ & $\begin{array}{c}31 \\
(32.97)\end{array}$ & $\begin{array}{c}26 \\
(27.65)\end{array}$ & 93.62 \\
\hline
\end{tabular}
genotypes with early leafing and late defoliation are damaged by late spring and early autumn frosts and yield decreases significantly. This study was aimed to identify new genotypes with both of late leafing and early defoliation characteristics and to reveal the relationships between main cultivar and F1 genotypes. The budburst time of the S-1/1 walnut genotype occurred on March 16, while the budburst time of the F1 genotypes changed between March 5 (Genotype 26-89) - April 26 (Genotype 21). The first leafing in main cultivar occurred on March 21, while the first leafing among F1 genotypes changed between March 15 (Genotype 89) and May 4 (Genotype 51). Leaf yellowing occurred on 4 November in the main genotype (S-1/1), while the time for leaf yellowing in other genotypes ranged from October 26 (Genotype 53) to November 21 (Genotype 93). The defoliation date in the main cultivar (S-1/1) occurred on 15 November, while it ranged from November 5 (Genotype 49) to December 18 (Genotype 67) in F1 genotypes. The studies carried out in different ecological regions of Turkey reported that genotypes and cultivars have leafing and flowering characteristics at different times (Asma et al. 1999; Tosun and Akçay 2005; Hassankhah et al. 2017; Akca et al. 2018; Orman 2018; Ozcan et al. 2020). Similar to the previous studies, phenological differences were determined between $S-1 / 1$ walnut genotype and the F1 genotypes. The differences in phenology among the genotypes can be attributed to genetic characteristics of the genotypes.

\subsection{Phenological data analysis}

169 Phenological observations are important in obtaining new varieties in fruit species. The results indicated that the 170 phenological diversity among walnut genotypes was significant (Table 3). Coefficient of variation values for 171 budburst, leafing, leaf yellowing and defoliation were calculated as 10.52, 9.32, 1.71 and 3.36, respectively 172 (Table 5). Descriptive statistics of the phenological traits for the walnut genotypes were given in Table 5. In a 173 similar study, Bukucu et al. (2020) reported PC1 as 50.226\%, PC2 as $36.635 \%$, and PC3 as $12.413 \%$ and PC4 174 as $0.726 \%$ in 684 walnut genotype. The $\mathrm{CV}$ value for the leafing date in 540 walnut genotypes was reported as $17577.78 \%$ by Khadivi-Khub et al. (2015). 
Table 5. Units, number of samples, maximum, minimum, mean, and standard deviation of phenological traits in 180 the walnut genotypes.

\begin{tabular}{cccccccc}
\hline Traits & Units & N & Min & Max & Mean \pm SD* & CV (\%) \\
\hline Budburst & Day & 95 & 63 & 115 & $78.55 \pm 8.26$ & 10.52 \\
\hline Leafing & Day & 95 & 73 & 123 & $87.62 \pm 8.17$ & 9.32 \\
\hline Leaf Yellowing & Day & 95 & 298 & 324 & $308.20 \pm 5.27$ & 1.71 \\
\hline Defoliation & Day & 95 & 308 & 351 & $329.05 \pm 11.07$ & 3.36 \\
\hline
\end{tabular}

181 *Standard Deviation (SD), Coefficient of Variance (CV\%)

182 The results of the Pears on correlation carried out to determine the relationships between phenological traits were

183 given in Figure 1. Significant positive correlation $(\mathrm{r}=0.8285)$ was obtained between budburst and leafing, and a

184 negative correlation was recorded between budburst and leaf yellowing and defoliation $(r=-0.0511, r=-$

$1850.0025)$.

186 The leafing had a positive correlation with leaf yellowing and defoliation $(r=0.0220, r=0.0436)$. The 187 defoliation had a positive correlation with leaf yellowing $(\mathrm{r}=0.5430)$. Similarly, Özcan et al. (2020) reported

188 positive correlation between leafing and defoliation $(r=0.0957)$, and Amiri et al. (2010) indicated similar 189 relationship between these two traits $(r=0.298)$. Significant correlations between leafing in walnut and other 190 phonologic traits have been reported also by others (Ebrahimi et al. 2015; Khadivi-Khub et al. 2015; Abedi and 191 Parvaneh 2016; Hassankhah et al. 2017; Bukucu et al. 2020).

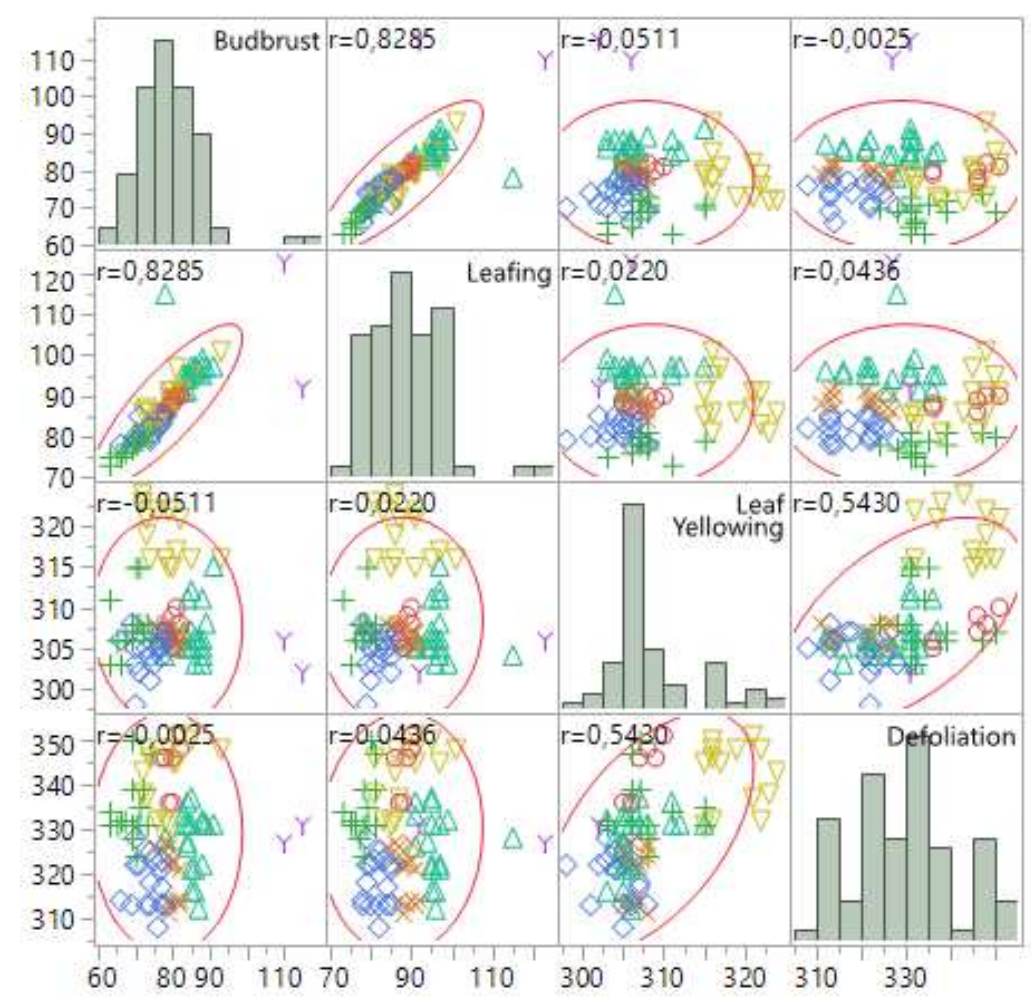

192

193 Figure 1. Scatter plot matrix and heatmap of correlations between phenological traits 
Principal component analysis (PCA) was carried out to determine overall differences among walnut genotypes. The first component (PC1) explains $45.739 \%$ of the total variation in phenological traits, the PC2 explains $38.646 \%$, PC3 $11.419 \%$ and PC4 $4.207 \%$. Bi-plot partially grouped walnut genotypes according to their parents (Table 6; Figure 2). Multivariate analysis is performed to summarize the variability of a complex data set and present the data in an interpretable form as in the principal components (Ribeiro et al. 2013). The purpose of PCA is to identify the main factors and influential parameters that distinguish participation (Khadivi-Khub et al. 2015). The PCA analyzes have been carried out for many plant traits in walnut, however, the number of PCA analyzes for phenological traits is limited (Cosmulescu and Trandafir 2011; Pop et al. 2013; Bou Abdallah et al. 2016; Arab et al. 2019).

204 Table 6. Eigenvectors of principal components (PC) for phenological traits in the walnut population

\begin{tabular}{ccccc}
\hline Traits & PC1 & PC2 & PC3 & PC4 \\
\hline Budburst & 0.706 & -0.045 & -0.015 & 0.706 \\
\hline Leafing & 0.707 & 0.016 & -0.051 & -0.705 \\
\hline Leaf Yellowing & -0.003 & 0.707 & 0.704 & 0.063 \\
\hline Defoliation & 0.032 & 0.707 & 0.704 & -0.063 \\
\hline \% of variance & 45.739 & 38.646 & 11.419 & 4.207 \\
\hline Cumulative variance & 45.739 & 84.375 & 95.789 & 100.00 \\
\hline
\end{tabular}
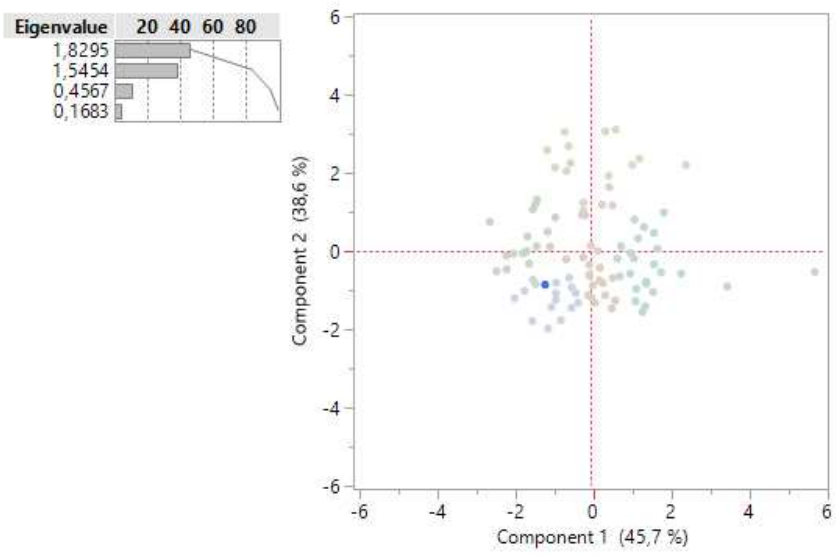

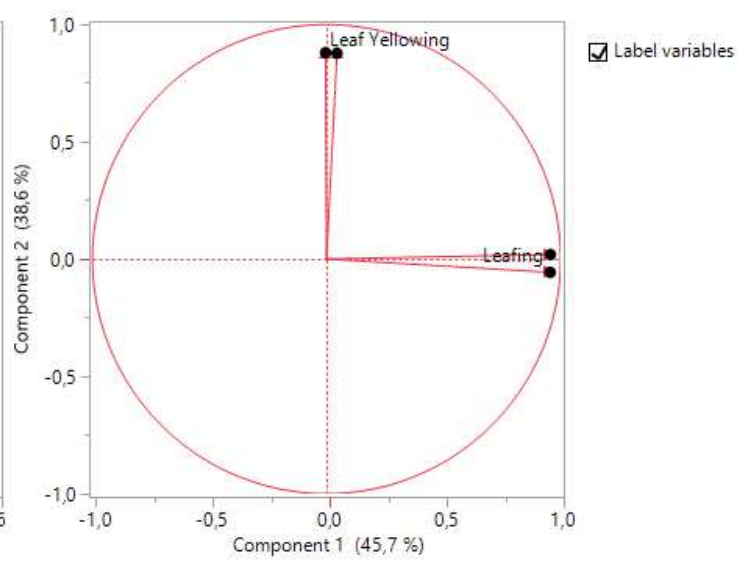

Figure 2 A) Scatter plot for the first two principal components for the walnut accessions based on phenological traits. B) Principal component analysis bi-plot of phenological traits among 94 walnut genotypes

210 Cluster analysis of the S-1/1 walnut genotype and 94 genotypes, including the seedlings from this genotype, was 211 performed to assess the relationship between walnut genotypes in a dendrogram based on 4 phenological traits

212 (Fig. 3). Walnuts were mainly split into 2 main clusters and 10 sub-clusters. The main cultivar (S-1/1) was 213 included in subset 5 of the first main cluster (Fig. 3).

214 The results of cluster analysis partially confirmed the genotype-related results obtained in PCA (Fig. 3). These 215 findings suggest that the walnuts are heterozygous, however, dominant genes largely determine the traits such as 216 leafing and defoliation dates. Morphological features have been used effectively to determine the genetic 217 variation among walnut genotypes (Arzani et al. 2008; Ebrahimi et al. 2010, 2011; Ghasemi et al. 2012; Norouzi 218 et al. 2013; Hussain et al. 2016; Cosmulescu and Stefanescu 2018; Rezaei et al. 2018; Bukucu et al. 2020). This 

pollinated walnut cultivars.

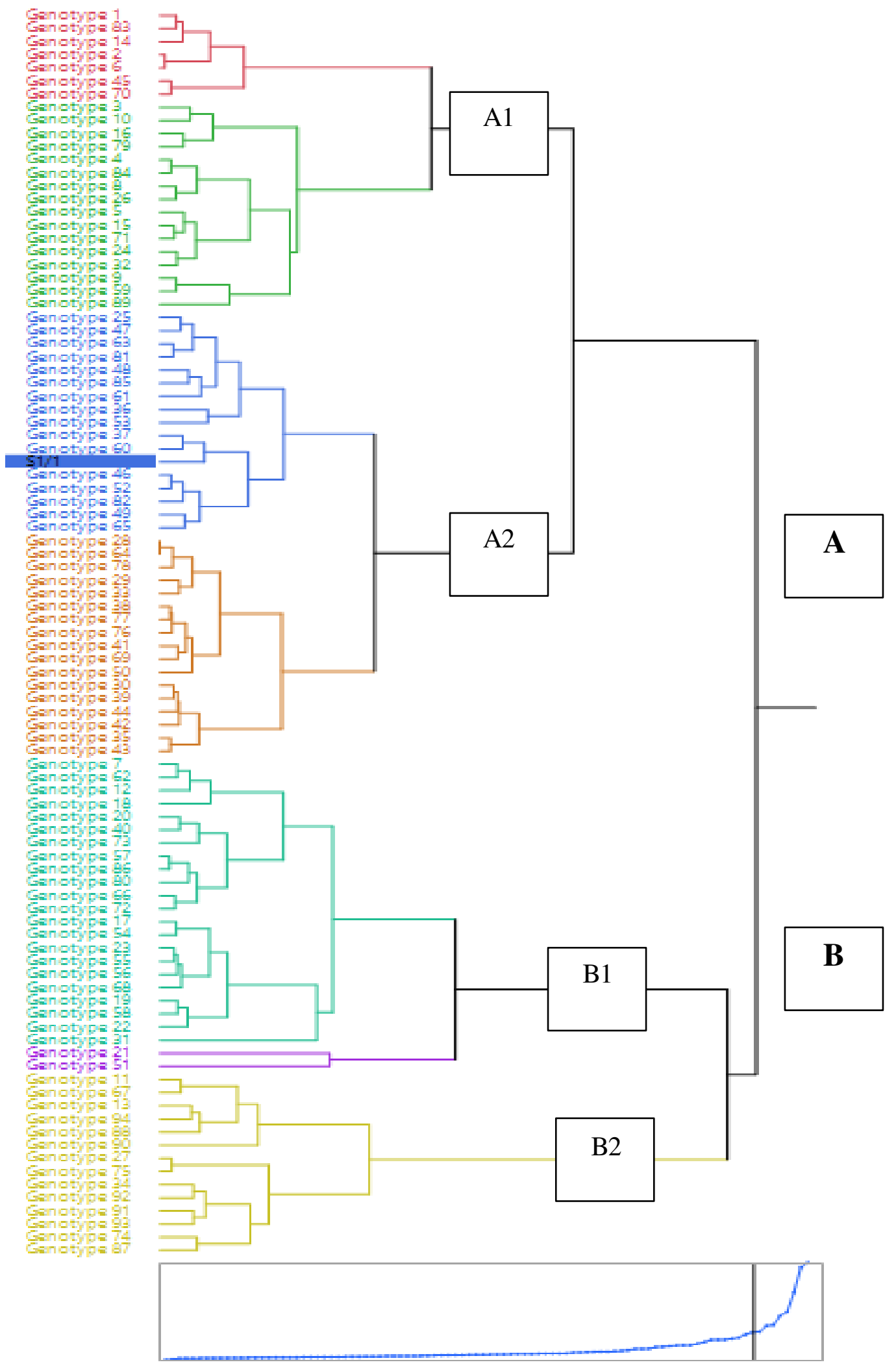

Figure 3 Phenotypic clustering of 94 walnut genotypes based on Ward's phenological pair-wise distance and phenological heat map. Cluster analysis of the genotypes is based on the traits with high heritability 


\subsection{Genetic Differences between Genotypes}

ISSR marker technique was used to determine the variation between the $S-1 / 1$ walnut genotype and the seedlings obtained from this genotype. Twelve ISSR primers were used in the study and the bands could not 227 obtain for 5 of the primers. Fifty two bands were obtained from 7 primers. Polymorphism was detected in 45 of 228 the 52 bands, while 7 bands were monomorphic (Figure 4).

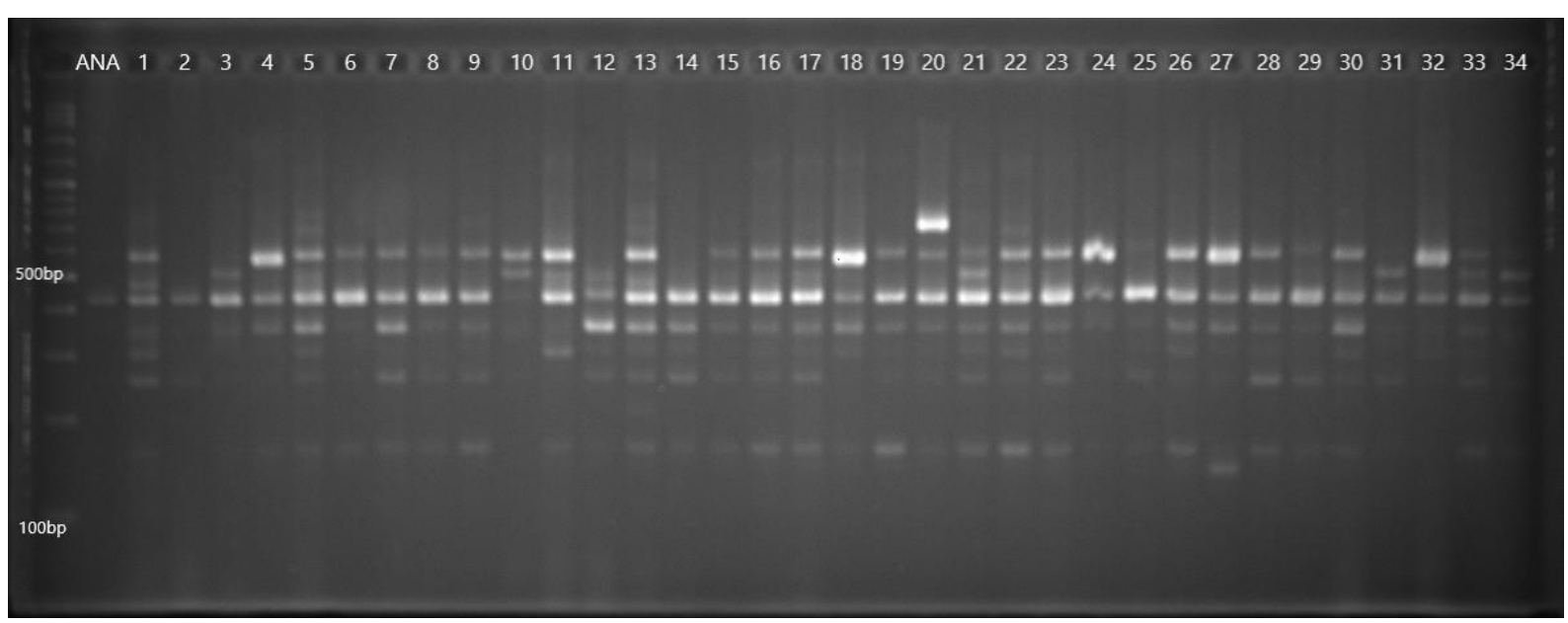

230 Figure 4. The appearance of the bands belonged to the ISP 5 primer combination

231 The polymorphism information content (PIC) of the primers used varied between 48 and $95 \%$. The 232 polymorphism rate of the ISSR primers used was $86.53 \%$. The primer that produced the most alleles (9) was 233 ISSR 5, and the average number of alleles was calculated as 7.42 (Table 7).

234 Table 7. Allele numbers and PIC (polymorphism information content) values of ISSR primers used in the study

\begin{tabular}{|c|c|c|c|c|}
\hline Number & $\begin{array}{c}\text { Name of } \\
\text { Primer }\end{array}$ & Base sequence & $\begin{array}{c}\text { Number of } \\
\text { Allele }\end{array}$ & PIC \\
\hline 1 & ISSR1 & CACACACACACAA & 5 & 0,63 \\
\hline 2 & ISSR4 & CACACACACACAGC & 4 & 0,94 \\
\hline 3 & ISSR5 & CACACACACACAG & 9 & 0,74 \\
\hline 4 & ISSR7 & ACACACACACACACACCG & 6 & 0,82 \\
\hline 5 & ISSR8 & ACACACACACACACACACC & 6 & 0,79 \\
\hline 6 & ISSR12 & GAGAGAGAGAGAGAGAGAC & 7 & 0,66 \\
\hline 7 & ISSR13 & AGAGAGAGAGAGAGAGC & 8 & 0,47 \\
\hline
\end{tabular}

236 Christopoulos et al. (2010) used the ISSR marker technique to determine the genetic differences and 237 relationships among 56 walnut genotypes in Greece. Pedigrees of 56 genotypes were revealed and many 238 international cultivars were grouped together with the walnut genotypes examined. Potter et al. (2002) 239 investigated the genetic relationships among 48 walnut (J. regia) cultivars in California using the ISSR 240 molecular marker technique. Eight polymorphic ISSR primers used in the study were selected after screening 47 
241 primers in four cultivars. Amplification of eight primers in 48 walnut cultivars yielded 54 bands and 31 (57\%)

242 of the bands indicated polymorphism. The number of bands per primer varied between 5 and 9 , and the number

243 of polymorphic bands varied between 1 and 7. The researchers have reported that the ISSR technique can be

244 used to identify walnut cultivars and genotypes and to determine the genetic relationships between them. In

245 addition, they stated that the ISSR technique has similar cost to RAPD but it has more polymorphism. Hu et al.

246 (2015) screened the polymorphism of genetic variation in 27 individuals belonging to the Juglans hopeiensis Hu

247 species from two different regions and identified 25 SSR primers. The number of alleles ranged from 2 to 9 ,

248 heterozygosity observed ranged from 0.016 to 0.933 (mean 0.468), and heterozygosity expected ranged from

2490.022 to 0.823 (mean 0.462). The researchers stated that the improvement of new SSR primers will be useful to

250 investigate the genetic structure of endemic species Juglans hopeiensis $\mathrm{Hu}$ and other Juglans species

251 populations.

\section{3.4. Dendrogram of the Genotypes}

253 The dendrogram of walnut genotypes was scanned with 7 ISSR markers, and the dendrogram genotypes 254 calculated using the NTSYSpc version 2.2 program with the UPGMA (unweighted pair group method with 255 arithmetic mean) method according to genetic distance matrix of Nei (1972) were divided into 2 main clusters. 256 Genetic similarity rates of walnut genotypes ranged from 0.52 to 0.93 . Seventy four genotypes were placed in 257 the same cluster (A) with the main genotype and the other 20 genotypes were in a separate cluster (B). Each of 258 two main clusters was divided into 2 intermediate clusters and 4 sub-clusters were formed. The results of cluster 259 analysis revealed that genotypes 85 and 86 had the closest similarity with $93.15 \%$ and genotype 22 with $90 \%$. 260 In addition, 14 genotypes (genotypes 9, 24, 25, 28, 30, 36, 37, 46, 47, 52, 61, 62, 65, 83) had both phenologic 261 and genetic similarities (Fig. 5).

262 Özcan et al. (2020) obtained 2 main and 2 subsets in the dendrogram formed using the UPGMA test for 91 263 genotypes obtained from Pedro variety. The similarity ratio was reported between 0.61 and 0.99 . The similarity 264 ratio of genotypes 44 and 64 was $100 \%$ and genotype 6 was more than $95 \%$. The results of crossbreeding 265 studies indicated that Pedro variety is an excellent parent that prioritizes late leafing. 


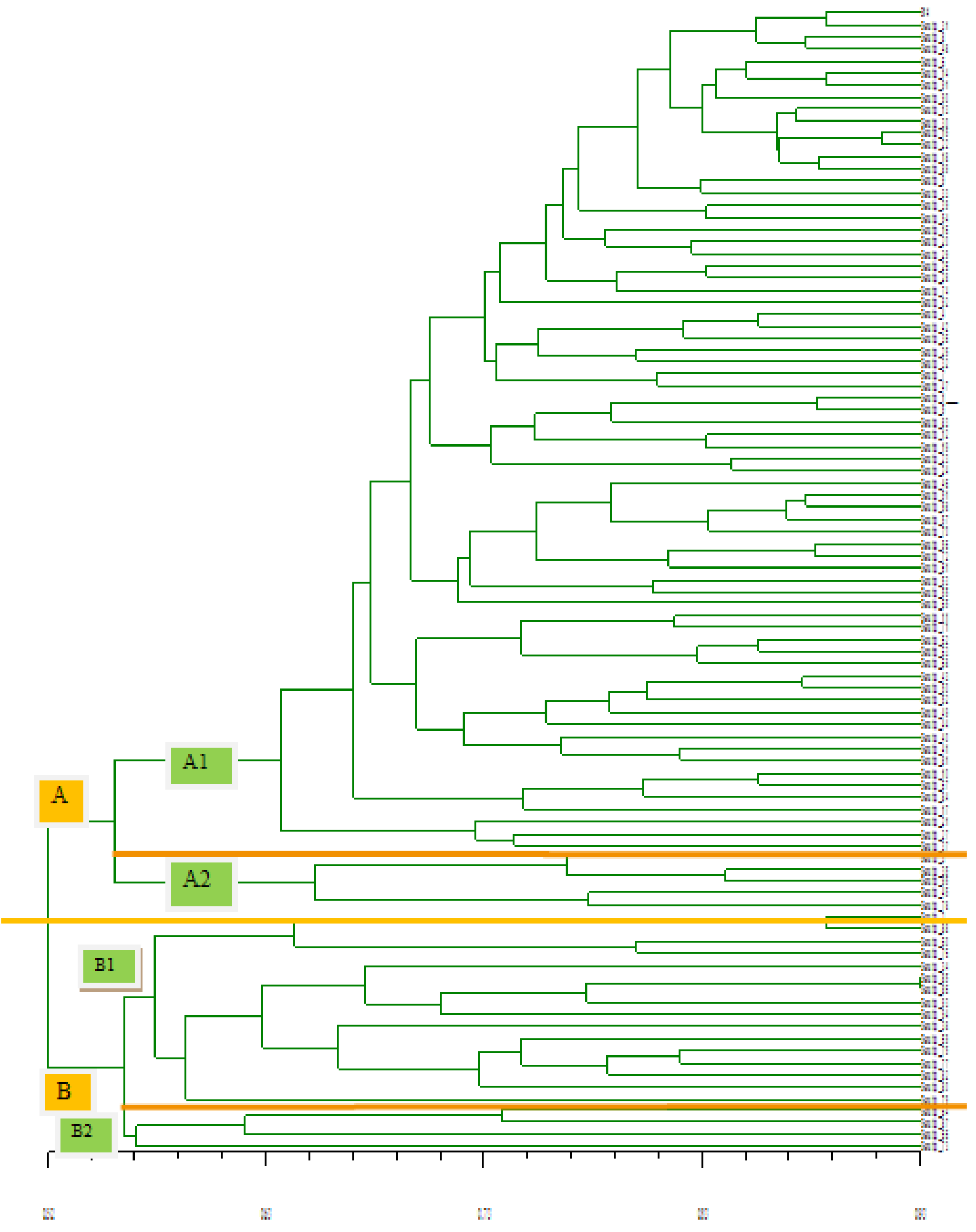

Figure 5. Genetic similarity dendrogram according to UPGMA method using ISSR data

\subsection{Genetic Structure Analysis of Population}

272 Cluster analysis was performed using Structure 2.3.4 software based on the Bayesian method to obtain 273 the optimum genetic group number of 94 walnut genotypes (Pritchard et al. 2000). The ideal group number of 94 
genotypes was determined by running the $\Delta \mathrm{K}$ values from 1 to 10 with 10 independent simulations for each $\Delta \mathrm{K}$ value. The permutation module was scanned in the range of 10,000-100,000 and repeated 5 times for each $\mathrm{K}$ value to determine the appropriate $\Delta \mathrm{K}$ value (Fig. 6). The results were stored in a zip file and uploaded to the 'Structure Harvester' program. The ideal $\Delta \mathrm{K}$ value was determined as 2. (http://taylor0.biology.ucla.edu/structureHarvester/).

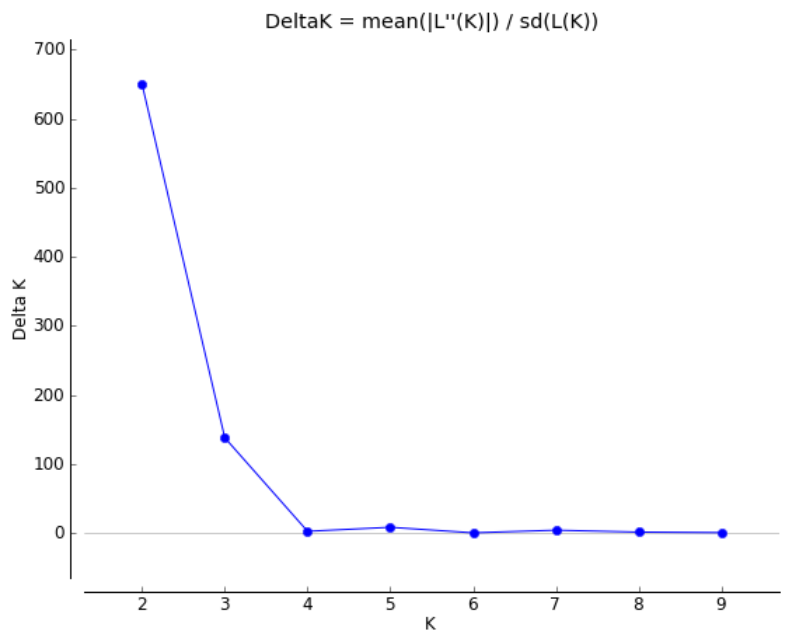

Figure 6. The ideal $\Delta \mathrm{K}$ value obtained from the Structure program

281 The diagram in Figure 7 was obtained by calculating the ideal K value obtained from the Structure 2.3 .4 282 program. Cluster analysis was carried out once more in Structure 2.3.4 program to determine the genetic 283 difference between genotypes. The cluster analysis indicated that genotypes were divided into 2 main groups. 284 Different colors in Figure 7 shows that the differences between genotypes. The genotypes close to the S-1/1 285 genotype were colored green and those farther away were colored red.
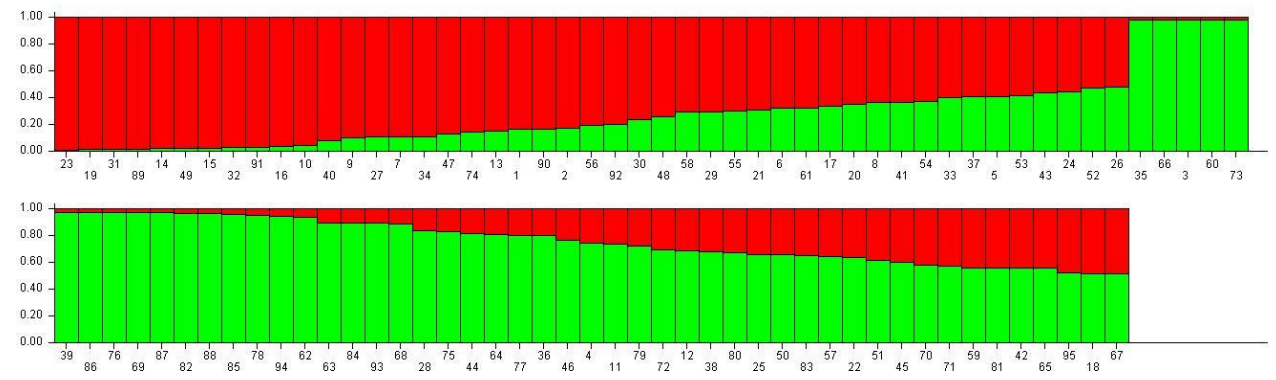

287 Figure 7. Cluster analysis obtained in Structure 2.3.4 program

\section{CONCLUSION}

290 The phenological and genetic similarity ratios between main parent genotype and 94 new genotypes obtained

291 from 200 open-pollinated seeds of S-1/1 walnut genotype were investigated. Important phenological differences 292 were recorded between the main genotype and F1 walnut genotypes. Average variation in phenological traits 293 among genotypes was $69.15 \%$, and the variation ratio in budburst, leafing, leaf yellowing and defoliation was $29475.54,73.41,34.05$ and $93.62 \%$, respectively. 
In molecular studies; 52 bands were obtained from 7 of 12 ISSR primers, and 45 of which were identified as polymorphic and 7 as monomorphic. Thus, the polymorphism was calculated as $86.53 \%$. Allele number was in monomorphic bands was calculated as 7.42. The PIC value ranged between 0.48 and 0.95 , and the average PIC value was calculated as 0.73 .

The dendrogram used to determine genetic variation indicated 2 main and 2 subsets. Seventy four genotypes were in the same cluster with the parent, and 20 genotypes were in the second cluster. The genetic similarity of walnut genotypes was between 52 and $93 \%$. The highest similarity rates were determined as $93 \%$ in 85 and 86 genotypes, and $90 \%$ in 15 and 22 genotypes. In addition, 14 genotypes (9, 24, 25, 28, 30, 36, 37, 46, 47, 52, 61, 62,65 , and 83 ) were both phenologically and genetically similar to the S-1/1 genotype. Significant variation at both phenological (69.15\%) and molecular level (52\%) in the seedling genotypes obtained from the S-1/1 genotype has important consequences for the future breeding programs. The results showed that evaluating the phenological and molecular findings together in the management of gene resources will provide a better characterization in breeding studies.

Conflicts of interest: The authors declare no conflict of interest

Authors contributions: Conceptualization, M.S. and S.B.; writing - original draft preparation, M.S., S.B., N.K., A.O., E.Y., B.S.B. A.B.; writing-review and editing, M.S., S.B., N.K., A.O., E.Y., B.S.B. A.B.; all authors contributed sections to the manuscript and approved the final version of the text.

\section{References}

Abedi B, Parvaneh T (2016) Study of correlations between horticultural traits and variables affecting kernel percentage of walnut (Juglans regia L.). J Nuts 7: 35-44

Ağaoğlu YS, Çelik H, M, Fidan Y, Gülşen Y, Günay A, Halloran N, Köksal Aİ, Yanmaz Y (2019) Genel Bahçe Bitkileri. Ankara Üniversitesi Ziraat Fakültesi Eğitim, Araştırma ve Geliştirme Vakfi Yayınları No: 4, Ankara s. 10- 11.

Amiri R, Vahdati K, Mohsenipoor S, Mozaffari MR, Leslie C (2010) Correlations between some horticultural traits in walnut. HortScience 45:1690-1694

Anonymous (1994) Walnut Descriptors for Walnut (Juglans spp.). International Plant Genetic Resources Institute, Rome, Italy.

Anderberg MR (1973) Cluster analysis for applications (No. OASTR- 73-9). Office of the assistant for study support kirtland AFBn mex

Arab MM, Marrano A, Abdollahi-Arpanahi R, Leslie CA, Cheng H, Neale DB, Vahdati K (2019) Combining phenotype, genotype and environment to uncover genetic components underlying water use efficiency in persian walnut. J Exp Bot 71:1107-1127 walnut (Juglans regia) genotypes from central Iran. N Zeal J Crop Hortic Sci 36:159-168 

Bahçe Bitkileri Kongresi, S:27-30, 14-17 Eylül, Ankara

Bardak A (2012) Lif ve hav oluşumuyla ilişkili DNA markörlerinin pamuk (Gossypium hirsutum L.) genomunda haritalanmas1 ve QTL analizi. Doktora Tezi. Kahramanmaraş Sütçü İmam Üniversitesi Fen Bilimleri Enstitüsü. Kahramanmaraş, s. 141.

Bardak A, Bölek Y (2012) Genetic diversity of diploid and tetraploid cottons determined by SSR and ISSR markers, Turkish Journal of Field Crops, 17(2): 139-144.

Bou Abdallah I, Baatour O, Mechrgui K, Herchi W, Albouchi A, Chalghoum A, Boukhchina S (2016) Essential oil composition of walnut tree (Juglans regia L.) leaves from Tunisia. J Essent Oil Res 28:545-550.

Bükücü ŞB, Özcan A, Sütyemez M, Ylldırm E (2020) Determination in the phenological difference levels of seedlings of some walnut genotypes (Juglans regia L.). Applied Ecology and Environmental Research 18(3):4807-4815.

Christopoulos MV, Rouskas D, Tsantili E, Bebeli PJ (2010) Germplasm diversity and genetic relationships among walnut (Juglans regia L.) cultivars and greek local selections revealed by inter-simple sequence repeat (ISSR) markers. Scientia Horticulturae, 125(4): 584-592.

Cosmulescu S, Trandafir I (2011) Variation of phenols content in walnut (Juglans regia L.). South West J Hortic Biol Environ 2:25-33

Doyle JJ, Doyle JL (1987) A Rapid DNA Isolation Procedure for Small Quantities of Fresh Leaf Tissue. Phytochemical Bulletin, 19:11-15.

Ebrahimi A, Fatahi MM, Zamani ZA, Vahdati K (2010) An investigation on genetic diversity of 608 Persian walnut accessions for screening of some genotypes of superior traits. Iran J Hortic Sci 40:83-94

Ebrahimi A, Fatahi R, Zamani Z (2011) Analysis of genetic diversity among some persian walnut genotypes (Juglans regia L.) using morphological traits and SSRs markers. Sci Hortic 130:146-151

Ebrahimi A, Khadivi-Khub A, Nosrati Z, Karimi R (2015) Identification of superior walnut (Juglans regia) genotypes with late leafing and high kernel quality in Iran. Sci Hortic 193:195-201

Filiz E, Koç İ (2011) Bitki biyoteknolojisinde moleküler markörler. Gaziosmanpaşa Üniversitesi Ziraat Fakültesi Dergisi, 2011(2).

Ghasemi M, Arzani K, Hassani D (2012) Evaluation and identification of walnut (Juglans regia L.) genotypes in Markazi province of Iran. Crop Breed J 2:119-124

Hassankhah A, Vahdati K, Rahemi M, Sarikhani Khorami S (2017) Persian walnut phenology: effect of chilling and heat requirements on bud break and flowering date. Int J Hort Sci Tech 4:259-271

Hu YH, Zhao P, Zhang Q, Wang Y, Gao XX, Zhang T, Zhou HJ, Dang M, Woeste KE (2015) De novo assembly and characterization of transcriptome using illumina sequencing and development of twenty five microsatellite markers for an endemic tree Juglans hopeiensis Hu in China. Biochem. Syst. Ecol., 63 : 201-211.

Hussain I, Sulatan A, Shinwari ZK, Raza G, Ahmed K (2016) Genetic diversity based on morphological traits in walnut (Juglans regia L.) landraces from Karakoram Region-I. Pak J Bot 48:653-659.

368 IPGRI (1994) Descriptors for walnut (Juglans spp.). International Plant Genetic Resources Institute, Rome

Khadivi-Khub A, Ebrahimi A, Sheibani F, Esmaeili A (2015) Phenological and pomological characterization of persian walnut to select promising trees. Euphytica 205:557-567 
Laborda PR, Oliveira KM, Garcia AA, Paterniani ME, De Souza AP (2005) Tropical maize germplasm: what can we say about its genetic diversity in the light of molecular markers. Thear. Appl. 111(7): 1288-1299

Nei M (1972) Genetic distance between populations. The American Naturalist, 106: 283-292.

Norouzi R, Heidari S, Asgari-Sarcheshmeh MA, Shahi-Garahlar A (2013) Estimation of phenotypical and morphological differentiation among some selected Persian walnut (Juglans regia L.) accessions. Intl J Agron Plant Prod 4:2438-2445.

Ozcan A, Sütyemez M, Nar A, Yıldırım E, Süslüoğlu Z (2020) Similarities of leafing and leaf fall date of 'Pedro' walnut variety with its progenies in breeding programs. Physiol Mol Biol Plants 26(10):19451959. https://doi.org/10.1007/s 12298-020-00872-5

Pop IF, Vicol AC, Botu M, Raica PA, Vahdati K, Pamfil D (2013) Relationships of walnut cultivars in a germplasm collection: comparative analys is of phenotypic and molecular data. Sci Hortic 153:124-135

Potter D, Gao F, Arello G, Leslie C, Mc Granahan GH (2002) Intersimple sequence repeat markers for fingerprinting and determining genetic relationships of walnut (Juglans regia) cultivars. J. Amer. Soc. Hort. Sci., 127(1):75-81.

Pritchard JK, Stephens M, Donnelly P (2000) Inference of population structure using multilocus genotype data. Genetics, 155(2):945-959.

Rezaei Z, Khadivi A, ValizadehKaji B, Abbasifar A (2018) The selection of superior walnut (Juglans regia L.) genotypes as revealed by morphological characterization. Euphytica 214:69. https://doi.org/10.1007/s 10681-018-2153-z

Ribeiro FADL, Mantovani GA, Poppi RJ, Rosa'rio FFD, Bezerra MCM, Bastos ALM, Melo VLAD (2013) PCA: Uma ferramenta para identificac,a o de trac,adores químicos para a'gua de formac,a a e a'gua de injec,a o $^{\circ}$ associadas a` produc,ão de petro'leo. Quim Nova 36:1281-1287.

Sutyemez M (2018) Bitki ıslahı ders notları (Yayınlanmamış). Kahramanmaras, Sütçü Imam Universitesi Ziraat Fakultesi Bahçe Bitkileri Bo“lümu“ BBB302

Sutyemez M, Özcan A, Bükücü ŞB(2018) Walnut cultivars through cross-creeding DİRİLiş and 15 TEMMUZ, The American Pomological Society, vol. 72, no. 3, pp. 173-180, Jul. 2018.

Tosun G, Akçay ME (2005) Yerli ve yabancı bazı ceviz geçișlerinin yalova ekolojisindeki fenolojik ve pomolojik özellikleri, Bahçe, Yalova Atatürk Bahçe Kültürleri Merkez Araştırma Enstitüsü Dergisi, II. Ulusal Ceviz Sempozyumu Özel Sayıs1, ISBN:1300-8943, S: 35-39, Yalova.

Ylldırım E, Sütyemez M (2020) Sütyemez-1 ceviz çeşidinden açık tozlanma ile elde edilen f1 bitkilerinin fenolojik ve moleküler karakterizasyonu. Yüzüncü Yıl Üniversitesi Tarım Bilimleri Dergisi 30(2): 299309

Yorgancılar M, Yakıșır E, Erkoyuncu MT (2015) Moleküler markörlerin bitki ıslahında kullanımı. Bahri Dağdaş Bitkisel Araştırma Dergisi, 4(2) : 1-12.

Zhang JF, Lu Y, Adragna H, Hughs E (2005) Genetic Improvement of New Mexico Acala Cotton Gerplasm and Their Genetic Diversity. Crop Science Doi: 10.2135/ cropsci 2005.0140

Zietkiewicz Ewa, Rafalski A, Damian L (1994) Genome fingerprinrting by simple sequence repeat (SSR)anchored polymerase chain reaction amplification . Genomics, 20(2):176-183. 